\title{
Shortcut ability in hamsters (Mesocricetus auratus): The role of environmental and kinesthetic information
}

\author{
NICOLE CHAPUIS \\ Université d'Aix-Marseille II, Marseille, France \\ and \\ PATRICIA SCARDIGLI \\ Centre National de la Recherche Scientifique, Marseille, France
}

\begin{abstract}
Shortcut ability in hamsters was tested in a complex wheel-shaped maze, in which kinesthetic information could be associated with environmental (proximal and/or distal) cues. The hamsters, previously trained to reach a hidden goal by a circumferential route, were led to the center of the maze to test their ability to take a directional shortcut to that same goal. In Experiment 1, their ability to accurately take a straight-line shortcut to the goal (from among eight possible paths) when distal cues were available was demonstrated. In Experiment 2, environmental information was neutralized in some conditions in order to enhance the relevance of kinesthetic information. When circumferential training runs were not too complex, the hamsters could use kinesthetic information-"path integration" processes-integrated during their previous training to infer the direction of the hidden goal.
\end{abstract}

The ability to take a shortcut or to negotiate a detour in a complex environment is likely to imply the use of a cognitive map of the environment, because both operations involve the building up of a relationship between several points in space in the absence of a direct stimulus. Piaget's developmental theory considered toddlers' ability to take detours and shortcuts as a criterion of the mastery of psychomotor development (Piaget, 1937). According to Piaget, this highest level of spatial construction, the level of the "representative group of displacements," allows children to succeed in detour and shortcut tasks in which the goal is hidden and in which the way to reach it needs to be represented. More recently, Pick and Rieser (1982) specified that cognitive mapping is implicated when the updating is done without the perception of all of the spatial relations; that is, "in an inferential way."

Tolman's definition of a "comprehensive map" (Tolman, 1948, pp. 192-193), and shortcut experiments done with rats (Gentry, Brown, \& Kaplan, 1947; Gentry, Brown, \& Lee, 1948; Kendler \& Gasser, 1948; Tolman, Ritchie, \& Kalish, 1946; Wilcoxon \& Waters, 1948) emphasized the role of shortcut ability as a process reflecting the building of a spatial representation. Unfortunately,

This research was supported by the Centre National de la Recherche Scientifique in Marseille, France. The authors especially thank F. Lavergne, G, Scotto, and D. Terramorsi for their assistance in conducting experiments, and M.-C. Buhot, M. Potegal, B. Poucet, and P. J. Urcuioli for their helpful comments on the manuscript. Address correspondence to N. Chapuis, Centre de Recherche de l'U.F.R.-S.T.A.P.S., 163 Avenue de Luminy, C.P. 910, 13288 Marseille Cedex 9, France. experimental conditions were not well controlled; the experiment by Tolman et al., for example, did not give any evidence for the rats' representation, because their orientation could be guided by a beacon-a light placed above the goal-thus indicating it from the choice point.

Three prerequisites must be met in order to demonstrate "true" shortcut ability. First, the goal should not be perceptible by the subject from the starting point. Second, the shortcut should consist of a new connection between two places. Third, its course should follow a straight line from the starting point to the goal, thus combining two aspects of orientation, distance, and direction, and optimizing them (least distance and zero angular deviation).

Menzel's experiments on cognitive mapping by apes were concerned with complex shortcut ability (Menzel, 1973). After the experimenter had shown the apes where various food items were hidden, the animals organized their subsequent search by reaching the goals by a path different from that used by the experimenter. They followed a least-distance strategy and therefore maximized the rate of food acquisition (Menzel, 1978). In this case, the three criteria for shortcut ability were met.

More recently, Chapuis and Varlet (1987) found that dogs (Alsatians) were able to take a shortcut in an outdoor situation after they had been given only one exploratory experience along two paths, DA and DB, which led to two baited points, A and B, hidden on the ground. When later released from point $\mathrm{D}$, the dogs were able to find the food in both places, taking the unexplored "directional shortcut" AB. Because the experiment was conducted outdoors, without marked paths, the results suggest 
that the dogs were able to select the direction of invisible goals from the whole range of possible directions by integrating motor and/or sensory cues collected during the earlier visit to these goals by the indirect way.

The present experiments with hamsters were conducted in much the same way as the experiment with dogs, but in laboratory conditions that allowed a more analytical approach. The animals were run in the alleys of a complex wheel-shaped maze in which kinesthetic information could be associated, or not, with environmental (proximal and/or distal) cues. The design involved training hamsters to run a particular circumferential route to a goalbox. This training was followed by a test in which they were allowed to choose, from a central choice point, the direct radial alley to the same goalbox (directional shortcut).

The main aim of this study was to answer two questions: (1) How accurate can a directional shortcut be? (2) Can animals calculate the distance and the angles between places and combine this information to infer the direction of one place from another on the basis of kinesthetic information, or do they need distal "place" cues in order to build up a relationship? Two shortcut experiments were conducted. The first experiment tested how accurate hamsters were in taking a directional shortcut. In the second experiment, the role of environmental and kinesthetic, or self-generated, information was studied.

A secondary purpose concerned how various orientation strategies might be used to solve a spatial task (O'Keefe \& Nadel, 1978; Schenk \& Morris, 1985; Sutherland, Chew, Baker, \& Linggard, 1987; Sutherland \& Dyck, 1984). As Whishaw and Mittleman (1986) and Sutherland et al. emphasize, several strategies can be used simultaneously in solving spatial problems, as is the case in natural surroundings (Chapuis, 1987). In the present experiments, it was necessary that a mapping strategy be used to reach the goal when the directional shortcut was chosen. However, if the animals did not take this direct route, they were allowed to run through the wheel-shaped maze using various pathways, thereby reaching the goal following other paths. Thus, a more precise analysis of the animals' spatial behavior could be worked out.

\section{EXPERIMENT 1}

\section{Method}

Subjects. Seven golden hamsters (Mesocricetus auratus), 4 females and 3 males approximately 60 days old at the start of the experiment, served as subjects. They were born and reared in the laboratory and were housed in standard cages with littermates of the same sex. The colony room was on a natural-light cycle. The animals were fed ad lib in their home cages, once a day after the experimental session, with hamster food pellets and pieces of carrot.

Apparatus. The apparatus was a wheel-shaped maze made of transparent Plexiglas, with eight radial alleys opening onto a central choice point. Eight circumferential alleys were linked together and with the radial alleys by eight boxes placed at the periphery. The maze was $140 \mathrm{~cm}$ in diameter; the walls of the alleys were $15 \mathrm{~cm}$ high.

The boxes were used as both starting boxes and goalboxes. For a given trial, all the boxes, except the starting box, contained an opaque cup; the reward (a mealworm) was hidden in the cup and placed in the box that was the present goal. The different sections of the maze (alleys, boxes, and central choice point) were separated by Plexiglas doors that were hinged at the top. These doors could be selectively locked so as to allow passage only in one direction, or could be completely unlocked to allow passage in both directions. Each box could be reached from either a radial or a circumferential alley. An electrical system monitored the locking and the unlocking of the doors.

The experimental room was visually heterogeneous, but no particular cue could directly indicate the position of the goal, because small cues (e.g., pictures on the wall) were always placed between two goalboxes and large cues (e.g., a set of shelves) were placed behind at least two goalboxes. Moreover, the choice point of the shortcut test was at a different place than the starting point and the paths of the previous training runs, so the view of the goal surroundings was different.

Procedure. The experiment consisted of seven successive experimental phases. Each phase was referred to as a "spatial problem," which involved the hamsters' taking a shortcut from the central choice point of the maze after having been trained to follow a particular circumferential path varying from one to seven successive segments of the wheel. The seven problems were labeled by the number of circumferential maze segments run in training, ranging from one segment (Problem P1) to seven segments (Problem P7), as depicted in Figure 1. For example, the clockwise training runs starting from box $A$ were paths $A B, A B C, A B C D, A B C D E$, ABCDEF, ABCDEFG, and ABCDEFGH for Problems P1, P2, P3, P4, P5, P6, and P7, respectively. Each subject was tested on all seven problems. The order and the direction (clockwise or counterclockwise) of the problems were counterbalanced across subjects.

Each experimental phase took place as follows. After having been trained to run to a goalbox from a starting box along a particular circumferential route for six daily sessions (training phase), a hamster was led to a central choice point where it was allowed to choose the direct pathway to the goalbox from among the radial alleys for one session (shortcut test).

Training phase. At the start of each daily training session, the animal was placed at the central choice point and was allowed to explore the maze for $10 \mathrm{~min}$ with all the doors unlocked. Then it was trained to follow a circumferential path (consisting of from one to seven segments) from a starting box to a goalbox, which was baited with a mealworm. The doors between the radial alleys and the circumferential path were closed, and the doors of the circumferential (outside) paths permitted passage in only one direction (i.e., from the starting box toward the goalbox).

After the hamster had eaten the mealworm, it was carried back to the starting box for another run. The hamsters were given five daily circumferential runs of the same number of segments over 6 days, for a total of 30 training trials.

Shortcut test. On Day 7 of each experimental phase, the subjects were given $10 \mathrm{~min}$ of exploration and two circumferential training runs, followed by three test trials. Each test trial consisted of forcing a hamster to run a detour to the center of the maze from the starting box by locking the two circumferential doors and unlocking the radial doors toward the center. When the hamster reached the center, the doors of each of the other radial alleys were unlocked. All the box doors were also unlocked so that the animal could reach the goal via any possible pathway, except the radial alley from which it came. The animals were rewarded in the goalbox on all five trials, as in the training phase.

Data collection. Three classes of responses were defined: (1) the directional shortcut, which consisted of taking the radial alley leading directly to the goal from the central choice point; (2) the "interception strategy," which consisted of reaching the circumferential paths by any other radial alley and then following the shorter circumferential path to the goal; and (3) incorrect responses-any response other than those of the first two classes. 

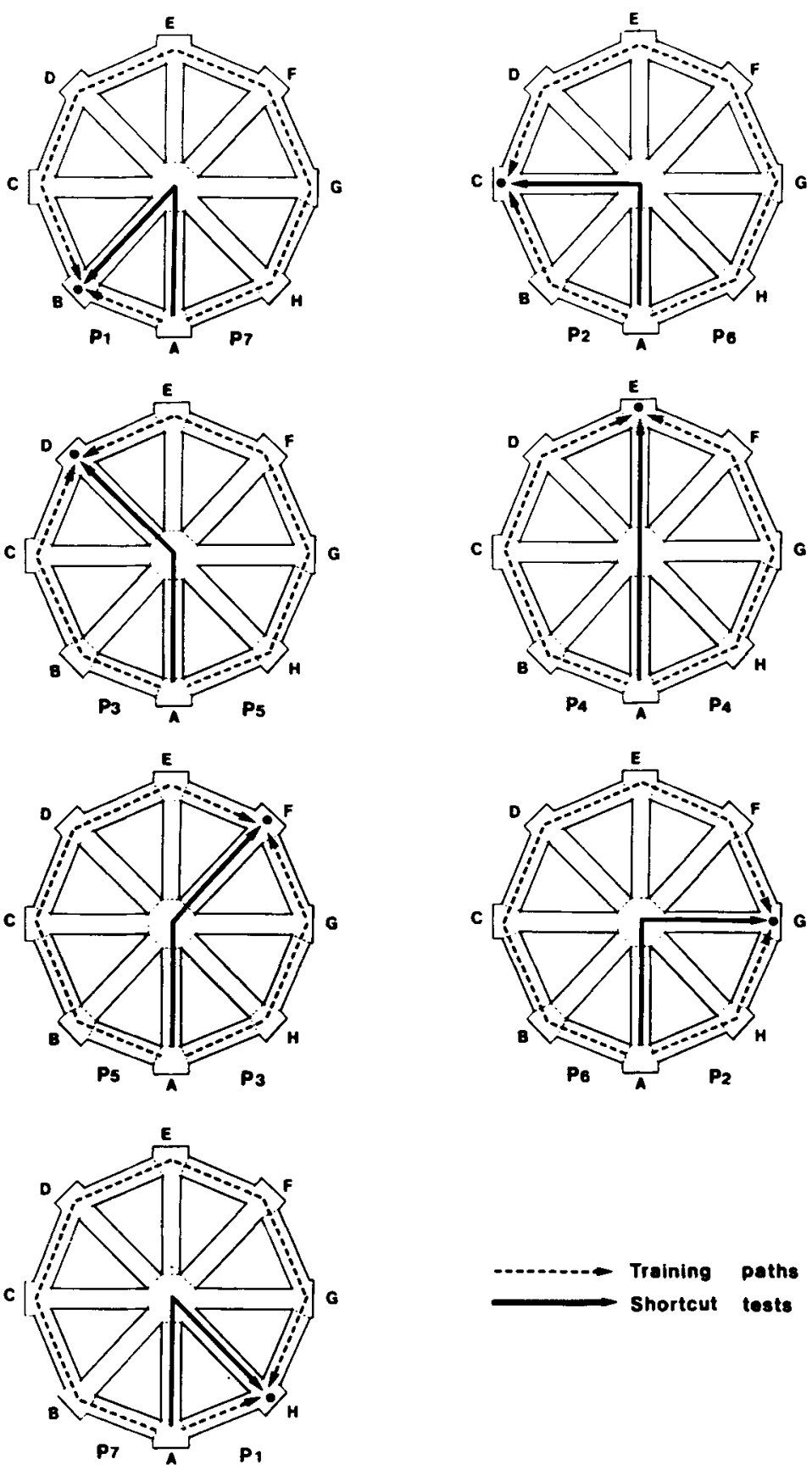

Figure 1. Maze diagrams of the seven spatial problems (P1-P7) used in Experiment 1 . Each diagram shows two problems, differentiated by whether the subjects were trained to run in either a clockwise or counterclockwise direction from the starting box (A). The circumferential path of each training phase is shown by the dotted lines. The solid lines indicate the choice of the directional shortcut taken from the central choice point of the maze during the shortcut tests following each training phase. The filled circles indicate baited cups placed in the goalhox. 

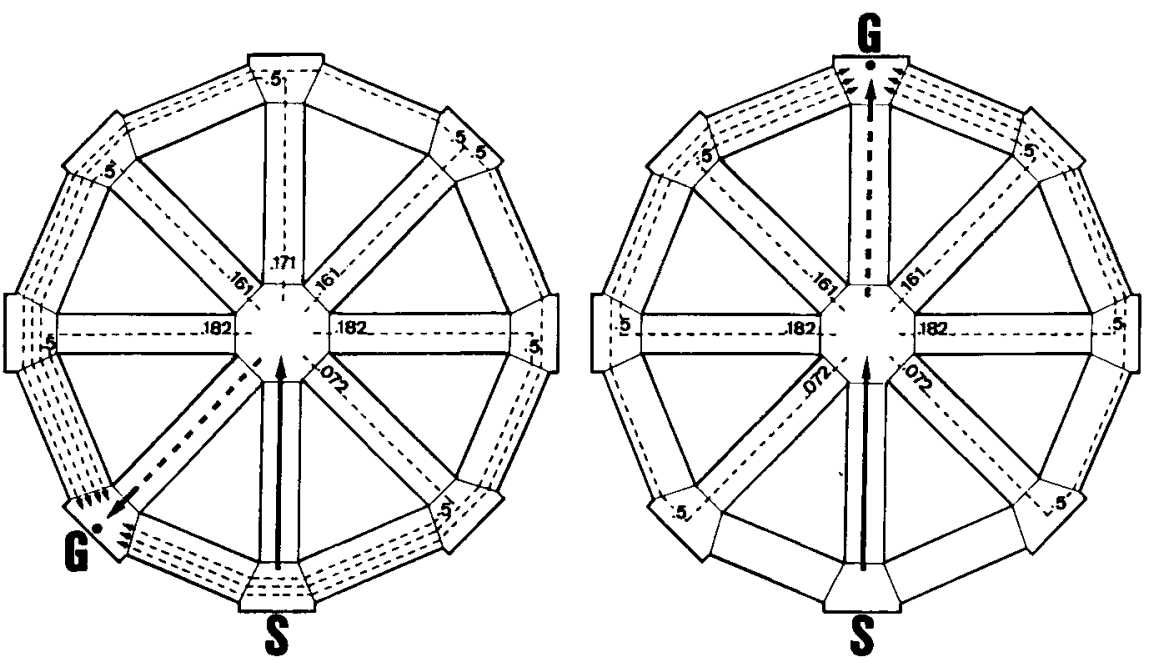

Figure 2. Example of the basis for estimating chance probabilities of occurrence of the interception strategy for Problems P1 and P7 (left maze) and P4 (right maze) in Experiment 1. The thick straight lines represent the starting path, the thick dotted lines indicate the directional shortcut, and the thin dotted lines show the interception strategy patterns. $S=$ starting box; $G=$ baited goalbox. See text for further details.

When the animal arrived at the central choice point during the shortcut test, it had to negotiate a particular angle to reach the goal directly. The correct turning angle was determined by the number of circumferential segments run during training. A turn corresponded to a rotation made by the animal between the arm it came from and the arm it entered. For one or seven segments, the angle was $135^{\circ}$; for two or six segments, it was $90^{\circ}$; for three or five segments, it was $45^{\circ}$; and for four segments, it was $0^{\circ}$. In order to establish a baseline probability of occurrence for each directional shortcut, the first three turns spontaneously made at the central choice point during the first seven exploratory phases of the experiment were recorded. The occurrence of the different turning angles $\left(45^{\circ}, 90^{\circ}\right.$, and $135^{\circ}$ clockwise and counterclockwise, and $\left.0^{\circ}\right)$ was compared with an equiprobability of selecting each of the seven alleys (i.e., $1 / 7=0.143$ ) and, later, with the directional shortcut performances in the shortcut tests. The frequency of occurrence of these baseline rotations was also taken into account in order to later analyze the choice of the interception strategy by the subjects during testing.

\section{Results}

Exploration. The probability of each turn at the central choice point during the exploratory phases was as follows: $p\left(0^{\circ}\right)=.171$ for angle $0^{\circ} ; p\left(45^{\circ}\right)=.161$ for angle $45^{\circ} ;$ $p\left(90^{\circ}\right)=.182$ for angle $90^{\circ}$, and $p\left(135^{\circ}\right)=.072$ for angle $135^{\circ}$, where the mean of right and left turns was used to calculate $45^{\circ}, 90^{\circ}$, and $135^{\circ}$ angle frequencies. Thus, $p\left(0^{\circ}\right)+\left[2 \times p\left(45^{\circ}\right)\right]+\left[2 \times p\left(90^{\circ}\right)\right]+[2 \times$ $\left.p\left(135^{\circ}\right)\right]=1$. Chi-square tests comparing these frequencies with an equiprobability of choice of each of the seven paths (i.e., .143) showed a significant difference for the $135^{\circ}$ angle $\left[\chi^{2}(1)=6, p<.02\right]$, which was chosen less often than by chance. By contrast, the probabilities of selecting for the $0^{\circ}, 45^{\circ}$, and $90^{\circ}$ angles did not differ significantly from chance $\left[\chi^{2}(1)=0.96,0.39\right.$, and 1.78 , n.s., respectively].
Orientation. The probability of the various directional shortcuts was compared with the baseline probabilities computed from the exploratory phases listed above (i.e., .072 for Problems P1 and P7; .182 for Problems P2 and P6; .161 for Problems P3 and P5; and .171 for Problem P4).

The rate of the interception strategy was computed by multiplying the same observed frequencies at the central choice point by .50 , as indicated in Figure 2 . The $.50 \mathrm{mul}$ tiplier assumes an equal probability of turning right or turning left at the first circumferential choice point. Only the first circumferential choice point was taken into account in order to simplify the analysis, and because very few subjects went back to the center from this point. Also, the .50 multiplier was not used for the circumferential path beginning at the box directly opposite to the goal; in this case, both paths to the goal were equivalent in length, so a probability of 1 was used instead. This latter computation did not apply to Problem P4, because the opposite path was the starting alley. The overall baseline probabilities for the interception strategy were as follows:

\section{For Problems PI and P7:}

$$
\begin{aligned}
\left(F 90^{\circ} \times 1 / 2\right)+\left(F 45^{\circ} \times 1 / 2\right)+\left(F 0^{\circ} \times 1 / 2\right)+\left(F 45^{\circ} \times 1\right) \\
+\left(F 90^{\circ} \times 1 / 2\right)+\left(F 135^{\circ} \times 1 / 2\right) \\
=\left[\left(F 0^{\circ}+F 135^{\circ}\right) \times 1 / 2\right]+\left(F 45^{\circ} \times 3 / 2\right)+F 90^{\circ} \\
=[(.171+.072) \times .5]+(.161 \times 1.5)+.182 \\
=.545 .
\end{aligned}
$$

For Problems P2 and P6:

$$
\begin{aligned}
\left(F 135^{\circ} \times 1 / 2\right)+\left(F 45^{\circ} \times 1 / 2\right)+\left(F 0^{\circ} \times 1 / 2\right)+\left(F 45^{\circ} \times 1 / 2\right) & +\left(F 90^{\circ} \times 1\right)+\left(F 135^{\circ} \times 1 / 2\right) \\
& +F 135^{\circ}+F 45^{\circ}+F 90^{\circ}+\left(F 0^{\circ} \times 1 / 2\right) \\
& =.072+.161+.182+(.171 \times .5) \\
& =.500 .
\end{aligned}
$$


For Problems P3 and P5:

$$
\begin{aligned}
\left(F 135^{\circ} \times 1 / 2\right)+\left(F 90^{\circ} \times 1 / 2\right)+ & +\left(F 0^{\circ} \times 1 / 2\right)+\left(F 45^{\circ} \times 1 / 2\right) \\
& +\left(F 90^{\circ} \times 1 / 2\right)+\left(F 135^{\circ} \times 1\right) \\
& =\left(F 135^{\circ} \times 3 / 2\right)+F 90^{\circ}+\left[\left(F 0^{\circ}+F 45^{\circ}\right) \times 1 / 2\right] \\
& =(.072 \times 1.5)+.182+[(.171+.161) \times .5] \\
& =.456 .
\end{aligned}
$$

For Problem P4:

$$
\begin{aligned}
& \left(F 135^{\circ} \times 1 / 2\right)+\left(F 9^{\circ} \times 1 / 2\right)+\left(F 45^{\circ} \times 1 / 2\right)+\left(F 45^{\circ} \times 1 / 2\right) \\
& \quad+\left(F 90^{\circ} \times 1 / 2\right)+\left(F 135^{\circ} \times 1 / 2\right) \\
& =F 135^{\circ}+F 90^{\circ}+F 45^{\circ} \\
& =.072+.182+.161 \\
& =.415 .
\end{aligned}
$$

Actual performances for each problem are presented in Figures 3 and 4 for the directional shortcut and interception strategy responses, respectively, along with their corresponding exploratory levels. Performances were calculated from a maximum of three responses per animal in both test and exploratory phases. The exploratory-level data points shown in these figures were derived by multiplying the baseline probability of each type of response by 3. For example, for Problems P2 and P6, the number of exploratory directional shortcut responses was $p\left(90^{\circ}\right)$ $\times 3=.182 \times 3=.546$. The corresponding figure for the interception strategy responses for these same two problems was $.500 \times 3=1.500$.

The curves presented in Figure 3 show an avoidance of directional shortcut paths placed at $135^{\circ}$ (i.e., for Problems P1 and P7) and a progressive decrease in the directional shortcut performances from Problems P2 to P6. Nonetheless, the level of this type of performance was well above chance (the exploratory level) for these latter problems. Figure 4 shows that the opposite trend, a continuous increase in the choice of the interception strategy, was observed for Problems P2 to P6, although it never exceeded chance level. However, the interception strategy

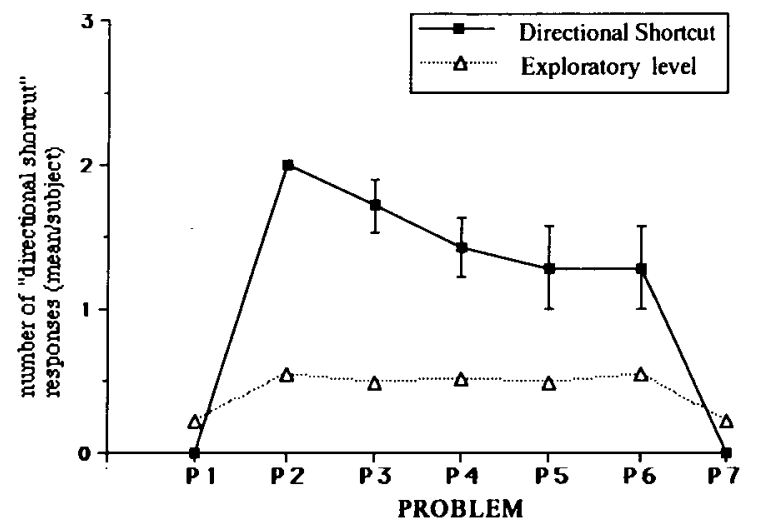

Figure 3. Directional shortcut performances for the seven problems, compared with their corresponding exploratory levels (number of responses averaged over subjects). The filled squares plot directional shortcut performances, and the open triangles plot exploratory levels.

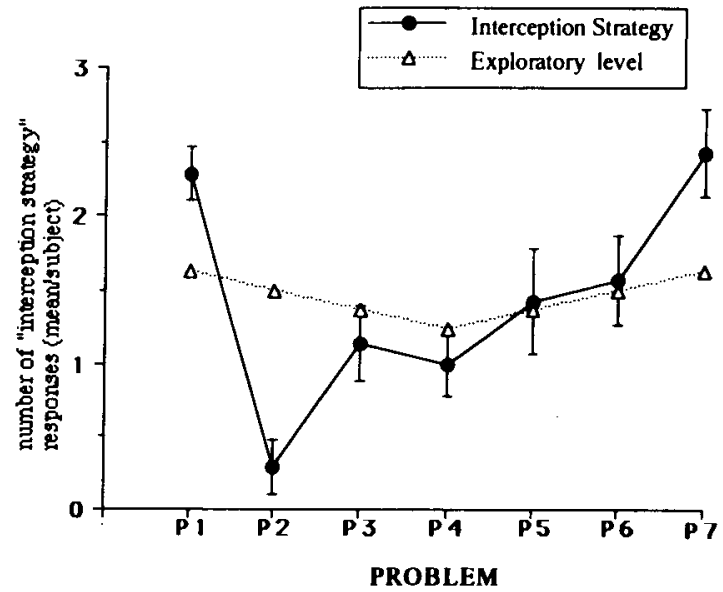

Figure 4. Interception strategy performances for the seven problems, compared with their corresponding exploratory levels (number of responses averaged over subjects). The filled circles plot interception strategy performances, and the open triangles plot exploratory levels.

was chosen more often than chance to solve Problems P1 and $P 7$, indicating that the animals were not actually lost.

Chi-square tests showed, on the one hand, that the choice of the directional shortcut was significantly above the exploratory level for Problems $P 2\left[\chi^{2}(1)=33.13\right.$, $p<.001] ; \mathrm{P} 3\left[\chi^{2}(1)=26.19, p<.001\right] ; \mathrm{P} 4\left[\chi^{2}(1)=\right.$ $13.80, p<.001] ;$ P5 $\left[\chi^{2}(1)=11.13, p<.001\right]$; and $\mathrm{P} 6\left[\chi^{2}(1)=8.58, p<.01\right]$. No calculation could be done for Problems P1 and P7 because there were no directional shortcut responses for these problems. On the other hand, the interception strategy was chosen more often than the exploratory level only for Problems $\mathrm{P} 1$ and $\mathrm{P} 7\left[\chi^{2}(1)=\right.$ $4.01, p<.05$, and $\chi^{2}(1)=5.96, p<.02$, respectively]. No significant differences were observed for Problems P3, P4, P5, or P6 $\left[\chi^{2}(1)=0.47,0.57,0.04\right.$, and 0.05 , respectively]. For Problem P2, the number of interception strategy choices was too low (2 trials out of 21$)$ to permit the analysis.

Finally, an analysis of variance (ANOVA) was performed to compare the directional shortcut performances across the seven problems (Rouanet \& Lépine, 1970). A main effect of problem was observed $[F(6,36)=16.22$, $p<.001]$. Paired comparisons showed significant differences when Problems P2, P3, P4, P5, and P6 were were compared with Problem P1 $[F(1,6)=\infty, p<.001$; $86.40, p<.001 ; 50.00, p<.001 ; 20.25, p<.01$, and $20.25, p<.01$, respectively $]$, and Problem P7 $[F(1,6)=$ $\infty, p<.001 ; 86.40, p<.001 ; 50.00, p<.001 ; 20.25$, $p<.01$, and $20.25, p<.01$, respectively], and also when Problem P2 was compared with Problems P4, P5, and P6 $[F(1,6)=8.00, p<.05 ; 6.25, p<.05 ; 6.25$, $p<.05$, respectively].

\section{Discussion}

The results of Experiment 1 showed that the hamsters were able to take accurate directional shortcuts in the task, 
except when the turning angle at the central choice point was obtuse, as in Problems P1 and P7. In this case, the animals preferentially used an interception strategy to reach the goal rather quickly. Comparison of these performances with the turns observed during the exploratory phases allowed a more precise estimate of this spatial ability. The hypothesis that the hamsters would avoid obtuse angles (in this case, $135^{\circ}$ ) during testing, for example, was suggested by the low frequency of choice of this angle during exploration.

Although the animals succeeded in the shortcut task for Problems P2 to P6, the number of directional shortcuts appeared to decrease progressively as the length of the circumferential path in training increased. This suggests that kinesthetic (self-generated vestibular, proprioceptive, and visual) information collected during previous training in the peripheral paths played a role in localizing the goal. Information was integrated during the animal's displacement, allowing it to infer the direction of the goal by another way. This is in agreement with Etienne, Teroni, Hurni, and Portenier's (1990) assessment that "spatial orientation implies the use of external cues as well as an "internal navigation" system which keeps track of the subject's locomotor progression"' (p. 17). According to the inertial guidance system described by Barlow (1964), which indicates that vestibular navigation is prone to cumulative errors, self-generated information would be less accurate as the distance run by the animal increases (cf. Potegal, 1982, 1987 for a review). Thus, long circumferential runs during training (e.g., for Problem P6) should yield less accurate goal localization in testing than would short circumferential runs (e.g., for Problem P2), as was observed.

Of course, the absence of directional shortcuts for Problems P1 and P7 cannot be due to a difficulty related to the length of the path alone because, in these two problems, the circumferential training runs were the shortest and the longest, respectively. As mentioned before, however, the results of these problems could simply be interpreted as a reluctance to make obtuse angles.

\section{EXPERIMENT 2}

In order to study more precisely the process of choosing the directional shortcut, the roles of environmental and kinesthetic information on shortcut ability were examined. For this purpose, situations were set up in which distal (extramaze) and proximal (intramaze) cues were both, or individually, made relevant or irrelevant. As in Experiment 1 , the effect of the previous training route on shortcut performances was also analyzed. However, the spatial problems that involved choice of the two obtuse angles at the central choice point were not tested, because the subjects avoided these obtuse-angle turns in Experiment 1.

\section{Method}

Subjects. Forty-one experimentally naive golden hamsters (Mesocricetus auratus), 21 females and 20 males approximately 60 days old at the start of the experiment, were used. They were reared and housed like the animals in Experiment 1.
Apparatus. The apparatus consisted of a steel wheel-shaped maze containing six radial alleys. Six circumferential alleys linked the six boxes at the ends of the radial alleys. Steel doors, which operated on the same principle as in the previous experiment, separated the different parts of the apparatus. Thus, the animals had a restricted view of the separate segments of the apparatus. The maze was $100 \mathrm{~cm}$ in diameter and the walls of the alleys were $20 \mathrm{~cm}$ high. It could be rotated on its central axis in order to manipulate the relevance of intra- and extramaze cues. It was located in the same, visually heterogeneous room that was used in Experiment 1 .

Procedure. Separate groups of hamsters were submitted to one of four experimental conditions in which the relevance of intramaze and extramaze cues was varied, as described below. The general procedure was identical for all conditions. Each group was successively submitted to three experimental phases corresponding to different spatial problems. A phase consisted of six training sessions and one shortcut test session, as described in Experiment 1. As shown in Figure 5, the three spatial problems corresponded to training in two, three, or four successive segments of the circumferential alleys (i.e., Problem P2, P3, or P4). These were given to each subject in a counterbalanced order. For each problem, half of the subjects ran in a clockwise direction, and the other half ran in a counterclockwise direction. The order and the direction of the training runs were varied in order to counterbalance the effects of the previous problems.

The role of kinesthetic information was tested as follows. In Condition Neither $(n=14)$, both intra- and extramaze information were made irrelevant; the starting boxes, the outside pathways, and the goalboxes were located at different places in the maze for consecutive trials. In this condition, only the "shape" of the outside path remained constant; that is, the length of the path (number of segments) and its direction (clockwise or counterclockwise) were identical across trials. The subjects could use only kinesthetic information while following its course.

Intramaze cues were made relevant in Condition Intra $(n=8)$. In this condition, the maze was rotated on its central axis $\left(60^{\circ}\right.$ in the same direction) on successive trials. For each trial, the subject "followed" the rotation of the maze in such a way that it was in the same part of the maze (same starting box, pathways, and goalbox), but not in the same place with regard to the room (thus preventing the use of extramaze cues).

Extramaze cues were made relevant in Condition Extra $(n=9)$. The maze was rotated in the same way as in Condition Intra, but the locations of the starting point, the paths, and the goal remained constant with respect to the experimental room. However, the parts of the apparatus used as starting points, paths, and goals varied across trials, thus neutralizing intramaze cues.

For Condition Both $(n=10)$, all cues were relevant. No change occurred in the outside path and, during each experimental session, the animal was run from and to the same place in terms of both the maze itself (intramaze cues) and the room (extramaze cues). This condition was equivalent to that used in Experiment 1 in terms of environmental features.

Data collection. The three classes of responses-directional shortcut, interception strategy, and incorrect responses-were defined in the same way as in Experiment 1.

Exploration rates obtained in Experiment 1 indicated that the rate of occurrence for the two obtuse turning angles was a little less than half the rate for the other five angles $(.42$, on the average). However, the two obtuse angles in the maze of this experiment were less obtuse than in Experiment $1\left(120^{\circ}\right.$ vs. $135^{\circ}$, respectively). With these considerations in mind, a theoretical chance rate of occurrence of .25 was assigned to each of the three acute turns $\left(60^{\circ}\right.$, $0^{\circ}, 60^{\circ}$ ) and of .125 to each of the two obtuse ones. Therefore, a .25 chance rate of occurrence of the directional shortcut was assumed for the three acute turns that would actually be tested.

As shown in Figure 6, the calculation of the chance rate of occurrence of the interception strategy was based upon the above fig- 


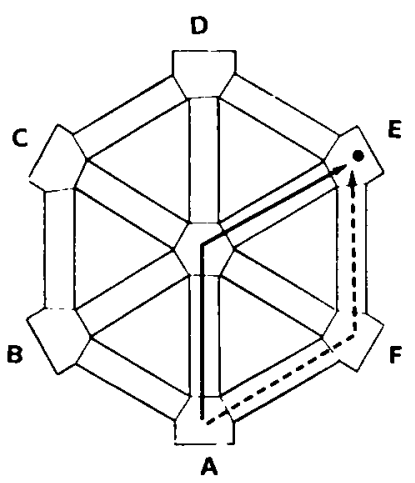

PROBLEM P2

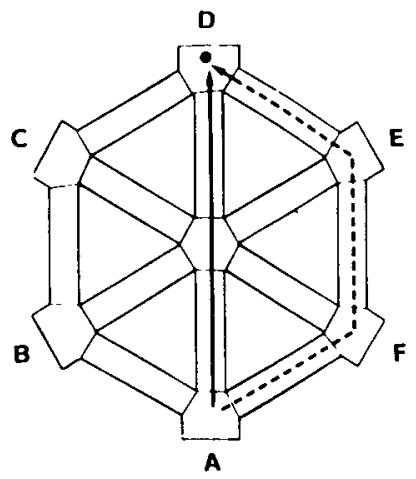

PROBLEM P3

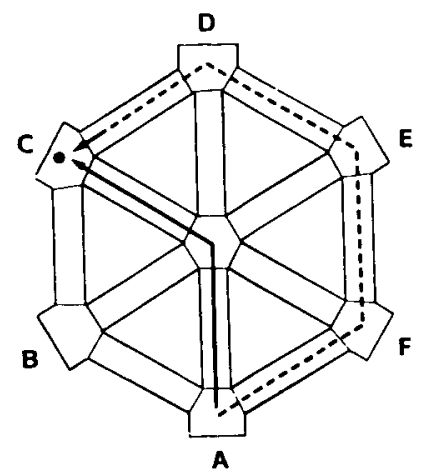

PROBLEM P4

Figure 5. Example of Problems P2, P3, and P4, used in Experiment 2. The dotted lines represent the training paths from the starting box (A) to the baited goal (filled circle). The straight lines indicate the choice of the directional shortcut taken from the central choice point of the maze.
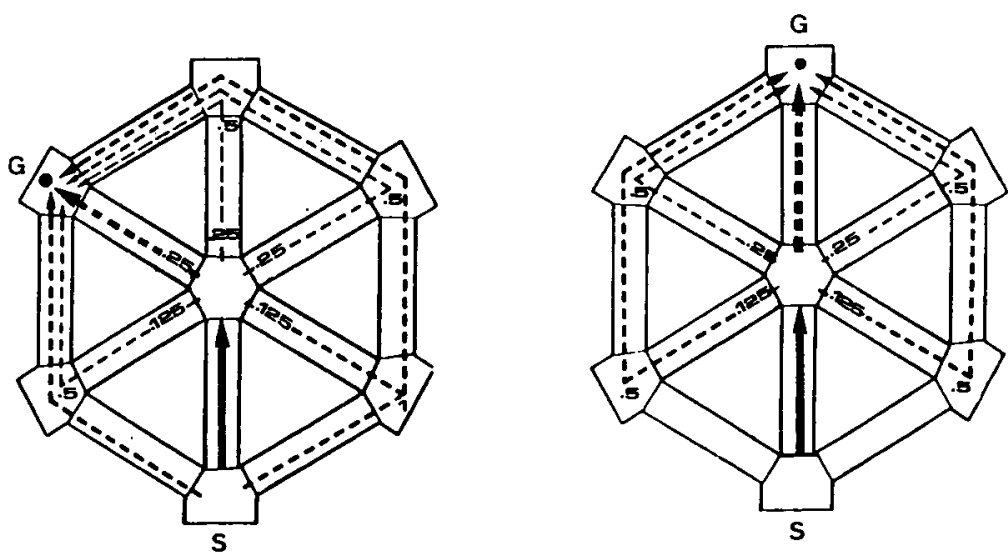

Figure 6. Basis for estimating chance probabilities of occurrence of the interception strategy for Problems P2 and P4 (left maze) and P3 (right maze) in Experiment 2. The thick straight lines represent the starting path, the thick dotted lines indicate the directional shortcut, and the thin dotted lines show the interception strategy patterns. $\mathbf{S}=$ starting box; $\mathbf{G}=$ baited goalbox. 
ures, and upon a .50 probability of taking the shorter peripheral path to the goal. As in Experiment 1, only the first circumferential choice point was taken into account. Also, the chance probability of .50 of reaching the goal by the shorter peripheral path was not used for the path opposite to the direct route to the goal; in this case, both paths were equivalent $(p=1)$. Again, the latter computation did not apply to Problem P3, because the opposite path was the starting alley. Thus, the estimated chance probability of the interception strategy for Problems P2 and P4 was $(2 \times .25 \times .5)$ $+(1 \times .125 \times .5)+(1 \times .125 \times 1)=.4375$. For Problem P3 it was $(2 \times .25 \times .5)+(2 \times .125 \times .5)=.375$.

\section{Results}

The number of directional shortcut and interception strategy responses for the three problems (P2, P3, and P4) are shown for each experimental condition in Figures 7 and 8, respectively. As in Experiment 1, performances were based upon a maximum of three responses. The exploratory (chance) level data points were thus calculated by multiplying the probability of each turn by 3 ; that is, $.25 \times 3=.75$, for the directional shortcut response in each of the three problems. For the interception strategy responses, the corresponding computations were $.4375 \times$ $3=1.312$ for Problems P2 and P4, and $.375 \times 3=1.125$ for Problem P3.

The results showed differences between groups for the directional shortcut performances. An ANOVA was performed to compare the rate of directional shortcuts between the experimental conditions. A main effect of condition, but not of problem, was found for directional shortcut performances $[F(3,37)=3.88, p<.025 ; F(2,74)=$ 1.12 , n.s., respectively]. No condition $\times$ problem interaction was observed $[F(6,74)=2.15$, n.s.]. Paired comparisons showed differences between Conditions Neither and Both $[F(1,22)=6.49, p<.025]$, Intra and Both $[F(1,16)=9.49, p<.01]$, and Intra and Extra $[F(1,15)=4.72, p<.05]$. An effect of condition was observed for Problems P2 and P4 $[F(3,37)=3.81, p<$ .025 , and $F(3,37)=3.00, p<.05$, respectively], but not for Problem P3 $[F(3,37)=0.89$, n.s. $]$.

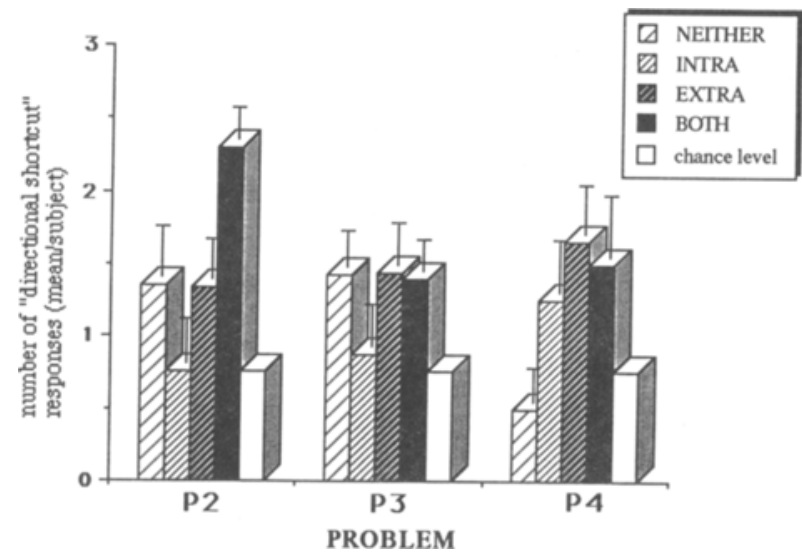

Figure 7. Directional shortcut performances (number of responses averaged over subjects) for each of the three problems in terms of environmental cues. Corresponding exploratory (chance) levels also are indicated.

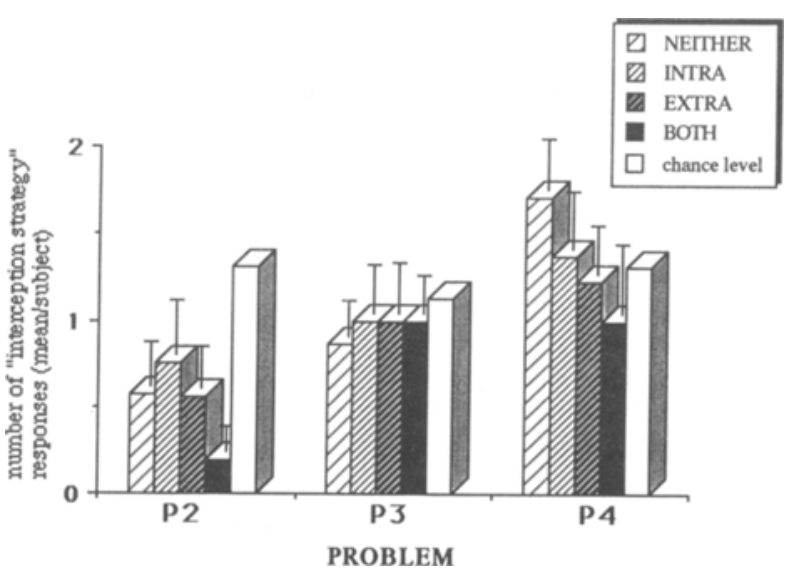

Figure 8. Interception strategy performances (number of responses averaged over subjects) for each of the three problems in terms of environmental cues. Corresponding exploratory (chance) levels also are indicated.

When performing the shortcut test, the hamsters chose the directional strategy in preference, except for Condition Intra. The observed use of directional shortcuts and interception strategies during testing was compared with their respective exploratory levels of occurrence by $\chi^{2}$ analyses. Averaged across problems, the directional shortcut strategy occurred significantly more often than chance in each condition, except for Condition Intra [for Condition Neither, $\chi^{2}(1)=8.89, p<.01$; for Condition Intra, $\chi^{2}(1)=1.85$, n.s., and for Conditions Extra and Both, $\chi^{2}(1)=25.68$ and 51.57, $p<.001$, respectively]. The use of the interception strategy did not differ from chance for Conditions Neither and Intra $\left[\chi^{2}(1)=2.36\right.$ and 1.43 , n.s., respectively]. For Conditions Extra and Both, it was used less often than chance $\left[\chi^{2}(1)=3.89, p<.05\right.$ and $10.98, p<.001$, respectively], although this result was simply a consequence of the fact that these subjects made many directional shortcut responses (thus limiting the number of possible interception responses).

Averaged over conditions, $\chi^{2}$ analyses also showed that directional shortcuts occurred more often than chance for all problems $\left[\chi^{2}(1)=32.20,16.07\right.$, and $11.45, p<$ .001 , for P2, P3, and P4, respectively]. Interception strategy performances were significantly different from chance only for Problem P2 [ $\left.\chi^{2}(1)=33.42, p<.001\right]$, where they were observed less often than expected by chance.

\section{Discussion}

The results from the conditions in which distal cues or all cues were available (Conditions Extra and Both) corroborate those obtained in Experiment 1. The data also indicate that the hamsters were able to take directional shortcuts in these tasks, even if they had no access to environmental cues (Condition Neither).

Curiously, the worst performances were observed in Condition Intra, and not in Condition Neither. In Condi- 
tion Intra, intramaze cues provided the only stable environmental information. This information could, for example, be olfactory cues deposited and collected in the circumferential alleys by the hamsters during training trials. However, this kind of intramaze cue would be difficult to perceive and localize from the central choice point. Also, local visual cues could have been collected during training; they too would be impossible to perceive from the choice point. So, like the group without available intra- and extramaze cues, the animals having only intramaze cues available also had no possibility of using these environmental cues at the central choice point to take the directional shortcut. Recent experiments have shown that hamsters are able to localize olfactory cues with reference to a stable spatial framework (Tomlinson \& Johnston, 1991). The present results do not disagree with these experiments, because the generally poorest performances of the intramaze cues group could be the result of a conflict between available kinesthetic information and unreliable exteroceptive information that could disturb them.

\section{GENERAL DISCUSSION}

The hamsters were able to accurately localize the place where they were previously fed and also reach a goal by a directional shortcut when they were required to deviate from their habitual route to this goal. Furthermore, they showed the ability to take shortcuts, even when only kinesthetic information was available. In other words, when no available environmental cue was given, the hamsters could infer the position of the goal by an integration of the distances and the angles experienced during the circumferential training paths. This ability seemed to be limited to simple paths, because the animals failed when the circumferential training paths consisted of running more than three segments and two angles. When distal information was relevant, the level of directional shortcut performances was higher. In this case, both experiments showed a progressive decrease in the frequency of the shortcut performances as the length of the training paths increased. This suggests that, even when external cues were available to localize the place of the goal, the animals took kinesthetic information into account. The use of an environmental referential framework would give them complementary information to localize the goal.

The use of kinesthetic information for taking a directional shortcut to reach a goal, after having previously reached it by a different way, is related to a "path integration" process, which implies that animals can encode angular and distance information in the course of their displacements. In particular, the vestibular system seems implicated in the process. According to the hypothesis of path integration, velocity signals from semicircular canals and otolithic end organs would be integrated in the central nervous system to provide information about angular and linear displacements from a zero point (cf. Potegal, 1982). Following Beritoff's (1965) work, a number of studies with animals have demonstrated that the vestibular system is involved in path integration (Chapuis, Krimm, de Waele, Vibert, \& Berthoz, 1992; Etienne, 1980; Etienne, Maurer, \& Saucy, 1988; Etienne, Teroni, Maurer, Portenier, \& Saucy, 1985; Horn, DeWitt, \& Nielson, 1981; Matthews, Ryu, \& Bockaneck, 1989; Miller, Potegal, \& Abraham, 1983; Mittelstaedt \& Mittelstaedt, 1980; Potegal, 1982, 1987; Semenov \& Bures, 1989). For example, Etienne et al. (1988) found that hamsters were able to compensate for passive rotations and, in some cases, passive linear displacements, in order to go back to their cages from the center of an arena. Lesions of the rat's vestibular system induced specific deficits in orientation during a return task requiring the evaluation of angles and distances following a passive outward transport (Matthews et al., 1989; Miller et al., 1983). Moreover, studies with humans have demonstrated specific roles for the semicircular canals in angular evaluation, and otoliths in distance evaluation (Berthoz, Israel, Vitte, \& Zee, 1988; Bloomberg, Melville-Jones, Segal, Mcfarlane, \& Soul, 1988; Israel \& Berthoz, 1989; Mittelstaedt \& Glasauer, 1991).

The improved performance when distal cues were available indicates that the hamsters were able to orient themselves to pinpoint a place by calculating the relation between a constellation of landmarks and a hidden goal. This agrees with data from other species of rodents by O'Keefe and Conway (1980), Morris (1981), and Collett, Cartwright, and Smith (1986), for example. Complementarity between environmental and self-generated information corroborates data from Etienne et al. (1990), obtained from a different spatial task using hamsters.

Concerning the choice of spatial strategies, an alternative use of directional shortcut and interception strategy is revealed by the results of the first experiment. In Experiment 1 , the rate of interception strategy increased progressively with the length of the peripheral paths, except for Problem P1. The interception strategy seemed preferentially used when kinesthetic information was too complex to give the precise location of the goal. In this case, the interception strategy would allow the animals to reach the goal easily. Nevertheless, for the one-segment problem (P1), a lack of directional shortcuts in relation to a significant use of the interception strategy was observed. As a matter of fact, the only two problems in which the hamsters failed to take the directional shortcut corresponded with a maximal turn at the choice point (135 ${ }^{\circ}$ angle, Problems P1 and P7). A tendency to avoid these angles was also observed during the previous exploratory phase of the maze. This can be interpreted as an avoidance of large angles, which is consistent with the "forward-going tendency" in complex mazes observed first by Dashiell and Bayroff (1931) and more recently by Bättig, Zahner, and Granjean (1964) and Uster, Bättig, and Nägeli (1976).

Note that angular factors are important parameters in orientation situations. This was the case in detour tasks studied by Chapuis, Thinus-Blanc, and Poucet (1983), Poucet, Thinus-Blanc, and Chapuis (1983), and Chapuis 
(1987). In these experiments with dogs, cats, and horses, the animals had to circumvent an obstacle. The arrangement of the obstacles (screens) was devised so as to leave two routes differing in their respective lengths (short and long) and/or angular deviation (narrow or wide) related to the starting-point/goal axis. The results suggested that the minimal angular deviation played a leading part in goal orientation and, in some cases, was preferred to the length factor.

The choice of the present experimental procedure, in which rodents were free to run all paths of the maze in order to reach the goal and were rewarded regardless of their performance, induced a variety of strategies in hamsters. Of course, this procedure cannot be viewed as more "natural" than a classical one, in which the reward is only associated with the choice of the optimal response; the wheel maze not being a natural environment for hamsters! Nevertheless, the interception strategy is analogous to the "inside shortcut" observed in some cases as an alternative to the directional shortcut in the experiment with dogs, described in the introduction, in which animals were free to organize their own route to the goal in a large meadow (cf. Chapuis \& Varlet, 1987).

This interception strategy was also observed in rats in an experiment we have designed using a similar wheel maze, but a different procedure (Buhot, Chapuis, Scardigli, \& Herrmann, 1991; Chapuis \& Herrmann, 1987). Rats with damage to the dorsal hippocampus were unable to find the goal by the direct way more often than chance. Nevertheless, they learned over time to travel over peripheral arms to reach the goal; that is, to use an interception strategy. In contrast, normal rats used this latter strategy at the same rate during the 24 daily sessions, whereas taking the direct way to the goal increased over time.

In summary, the use of the interception strategy is a viable alternative when there is not enough information available, when information is too complex to be integrated (e.g., for hippocampus-damaged animals), or when directional shortcuts involve taking obtuse angles. In these experiments, it was demonstrated that the simultaneous use of various spatial strategies can solve complex spatial problems.

\section{REFERENCES}

BARLOW, J. S. (1964). Inertial navigation as a basis for animal navigation. Journal of Theoretical Biology, 6, 76-117.

BätTIG, K., ZahNer, H., \& Granjean, E. (1964). Untersuchungen über die spontane Alternation der Ratte. Zeitschrifi für Experimentelle und Angewandte Psychologie, 11, 1-26.

BERITOFF, J. S. (1965). Neural mechanisms of higher vertebrates (W. Liberson, Trans.). Boston: Little, Brown.

Berthoz, A., Israel, I., Vitte, E., \& ZeE, D. (1988). Linear displacement can be derived from otolithic information and stored on spatial maps controlling the saccadic system. Advances in Oto-RhinoLaryngology, 41, 76-81.

Bloomberg, J., Melville-Jones, G., Segal, B., McFarlane, S., \& Soul, J. (1988). Vestibular-contingent voluntary saccades based on cognitive estimates of remembered vestibular information. Advances in Oto-Rhino-Laryngology, 41, 71-75.

Buhot, M. C., Chapuis, N., Scardigli, P., \& Herrmann, T. (1991). Spatial problem-solving in a wheel-shaped maze: Quantitative and qualitative analyses of the behavioural changes following damage to the hippocampus in the rat. Behavioural Brain Research, 44, 67-79.

Chapuis, N. (1987). Detour and shortcut abilities in several species of mammals. In P. Ellen \& C. Thinus-Blanc (Eds.), Cognitive processes and spatial orientation in animal and man (Vol. 1, pp. 97-106). Dordrecht: Martinus Nijhoff.

Chapuis, N., \& Herrmann, T. (1987). Spatial problem solving by rats with hippocampal lesions. Society for Neuroscience Abstracts, 13, 1319.

Chapuis, N., Krimm, M., De Waele, C., Vibert, N., \& BerTHOZ, A. (1992). Effect of post-training unilateral labyrinthectomy in a spatial orientation task by guinea pigs. Behavioural Brain Research, 51, 115-126.

Chapuis, N., Thinus-Blanc, C., \& Poucet, B. (1983). Dissociation of mechanisms involved in dogs' oriented displacements. Quarterly Journal of Experimental Psychology, 35B, 213-219.

Chapuis, N., \& Varlet, C. (1987). Shortcuts by dogs in natural surroundings. Quarterly Journal of Experimental Psychology, 39B, 49-64.

Collett, T. S., CARTwright, B. A., \& Smith, B. A. (1986). Landmark learning and visuo-spatial memories in gerbils. Journal of Comparative Physiology A, 158, 835-851.

DASHIELl, J. F., \& BAYRofF, A. G. (1931). A forward going tendency in maze running. Journal of Comparative Psychology, 12, 77-94.

EtienNe, A. S. (1980). The orientation of the golden hamster to its nest site after the elimination of various sensory cues. Experientia, 36, 1048-1050.

Etienne, A. S., Maurer, R., \& Saucy, F. (1988). Limitations in the assessment of path dependent information. Behaviour, 106, 81-111.

Etienne, A. S., Teroni, E., Hurni, C., \& Portenier, V. (1990). The effect of a single light cue on homing behaviour of the golden hamster. Animal Behaviour, 39, 17-41.

Etienne, A. S., Teroni, E., Maurer, R., Portenier, V., \& SAUCY, F. (1985). Short distance homing in a small mammal: The role of exteroceptive cues and path integration. Experientia, 41, 122-125.

Gentry, G., Brown, W. L., \& Kaplan, S. L. (1947). An experimental analysis of the spatial location hypothesis in learning. Journal of Comparative \& Physiological Psychology, 40, 309-312.

Gentry, G., Brown, W. L., \& LeE, H. (1948). Spatial location in the learning of a multiple T-maze. Journal of Comparative \& Physiological Psychology, 41, 312-318.

Horn, K. M., DeWitt, J. R., Nielson, H. C. (1981). Behavioral assessment of sodium arsanilate induced vestibular dysfunction in rats. Physiological Psychology, 9, 371-378.

IsRael, I., Berthoz, A. (1989). Contribution of the otoliths to the calculation of linear head displacement. Jourmal of Neurophysiology, 62, 247-263.

Kendler, H. H., \& GASSER, W. P. (1948). Variables in spatial learning: 1. Number of reinforcements during training. Journal of Comparative \& Physiological Psychology, 41, 178-187.

Matthews, B. L., Ryu, J. H., \& Bockaneck, C. (1989). Vestibular contribution to spatial orientation: Evidence of vestibular navigation in an animal model. Acta Otolaryngologica, 468, 149-154.

MEnzel, E. W. (1973). Chimpanzee spatial memory organization. Science, 182, 943-945.

MENZEL, E. W. (1978). Cognitive mapping in chimpanzees. In S. H. Hulse, H. Fowler, \& W. K. Honig (Eds.), Cognitive processes in animal behavior (pp. 375-422). Hillsdale, NJ: Erlbaum.

Miller, S., Potegal, M., \& Abraham, L. (1983). Vestibular involvement in a passive transport and return task. Physiological Psychology, 11, 1-10.

Mittelstaedt, M. L., \& Glasauer, S. (1991). Idiothetic navigation in gerbils and humans. Zoologische Jahrbücher. Abteilung für Allgemeine Zoologie und Physiologie der Tiere, 95, 427-435. 
Mittelstaedt, M. L., \& Mittelstaedt, H. (1980). Homing by path integration in a mammal. Naturwissenschafien, 67, 566-567.

Morris, R. G. M. (1981). Spatial localization does not require the presence of local cues. Learning \& Motivation, 12, 239-260.

O'KeEFE, J., \& CONWAY, D. H. (1980). On the trail of the hippocampal engram. Physiological Psychology, 8, 229-238.

O'KeEFE, J., NADEL, L. (1978). Hippocampus as a cognitive map. Oxford University Press.

Piaget, J. (1937). La construction du réel chez l'enfant. Neuchatel: Delachaux et Niestlé.

PICK, H. L., \& RiESER, J. J. (1982). Children's cognitive mapping. In M. Potegal (Ed.), Spatial abilities: Development and physiological foundations (pp. 107-128). New York: Academic Press.

Potegal, M. (1982). Vestibular and neostriatal contributions to spatial orientation. In M. Potegal (Ed.), Spatial abilities: Development and physiological foundations (pp. 361-387). New York: Academic Press.

Potegal, M. (1987). The vestibular navigation hypothesis: A progress report. In $\mathrm{P}$. Ellen \& C. Thinus-Blanc (Eds.), Cognitive processes and spatial orientation in animal and man (Vol. 2, pp. 28-34). Dordrecht: Martinus Nijhoff.

Poucet, B., Thinus-Blanc, C., \& Chapuis, N. (1983). Route-planning in cats, in relation to the visibility of the goal. Animal Behaviour, 31, 594-599.

RouANET, H., \& LEPINE, D. (1970). Comparisons between treatments in a repeated measurement design: ANOVA and multivariate methods. British Joumal of Mathematical \& Statistical Psychology, 23, 147-163.

SCHENK, F., \& MORRIS, R. G. M. (1985). Dissociation between components of spatial memory in rats after recovery from the effect of retrohippocampal lesions. Experimental Brain Research, 58, 11-28.
Semenov, L. V., \& Bures, J. (1989). Vestibular stimulation disrupts acquisition of place navigation in the Morris water tank task. Behavioral \& Neural Biology, 51, 346-363.

Sutherland, R. J., Chew, G. L., Baker, J. C., \& Linggard, R. C. (1987). Some limitations on the use of distal cues in place navigation by rats. Psychobiology, 15, 48-57.

Sutherland, R. J., \& DYCK, R. H. (1984). Place navigation by rats in a swimming pool. Canadian Journal of Psychology, 38, 322-347.

Tolman, E. C. (1948). Cognitive maps in rats and men. Psychological Review, 55, 189-208.

Tolman, E. C., Ritchie, B. F., \& Kalish, D. (1946). Studies in spatial learning: 1. Orientation and the short-cut. Journal of Experimental Psychology, 36, 13-24.

Tomunson, W. T., \& Johnston, T. D. (1991). Hamsters remember spatial information derived from olfactory cues. Animal Learning \& Behavior, 19, 185-190.

USTER, H. J., BätTIG, K., \& NäGELI, H. H. (1976). Effects of maze geometry and experience on exploratory behavior in the rat. Animal Learning \& Behavior, 4, 84-88.

Whishaw, I. Q., \& Mittleman, G. (1986). Visits to starts, routes, and places by rats (rattus norvegicus) in swimming pool navigation tasks. Journal of Comparative Psychology, 100, 422-431.

WILCOXON, H. C., \& WATERS, R. H. (1948). Spatial orientation in the white rat. Journal of Experimental Psychology, 38, 412-419.

(Manuscript received August 6, 1991; revision accepted for publication January 8, 1993.) 